\title{
Numerical Simulation on the Device of Rare Earth Oxide Preparation by Rare Earth Chloride Spray Pyrolysis
}

\author{
Yan Liu, ${ }^{1}$ Xiaolong Li, ${ }^{1}$ Xiangli Nan, ${ }^{2}$ Chao Lv, ${ }^{1}$ Chen Zhang, ${ }^{1}$ and Ting' an Zhang ${ }^{1}$ \\ ${ }^{1}$ Key Laboratory of Ecological Utilization of Multi-Metal Intergrown Ores of Ministry of Education and School of \\ Materials and Metallurgy, Northeastern University, Shenyang 110819, China \\ ${ }^{2}$ Environmental Resources, Materials and Metallurgy, Northeastern University, Shenyang 110819, China
}

Correspondence should be addressed to Yan Liu; liuyan@smm.neu.edu.cn

Received 16 March 2015; Revised 23 June 2015; Accepted 2 July 2015

Academic Editor: Vassilios C. Loukopoulos

Copyright (C) 2015 Yan Liu et al. This is an open access article distributed under the Creative Commons Attribution License, which permits unrestricted use, distribution, and reproduction in any medium, provided the original work is properly cited.

\begin{abstract}
Based on the industrial applications of jet pyrolysis technology and it being used for the production of rare earth oxides, the paper hopes to find out an efficient, economic, and environmental friendly new technology which is suitable for production of rare earth oxides. In this paper, a chloride pyrolysis water model is designed, and standard $\kappa-\varepsilon$ turbulence model and VOF model were coupled to simulate the three-dimensional steady gas-liquid flow in jet reactor. The valuable parameter we got provides strong basis for the experimental equipment manufacturing in thermal state and determining test program. Conclusions show that when the drainage tube diameter is $3 \mathrm{~mm}$, it can guarantee that two-phase distribution of the gas-liquid is more uniform and easy for gas-liquid mixing in the tail region of the Venturi tube. If the fluid near the middle of the duct in front of the Venturi starts to reach equilibrium as constant speed flowing, it proves that elongated pipe is conducive to obtain a stable flow of air required by experiments. In the adjustable pipeline location of the Venturi tube, fluid can form a closed loop and generate reflux in the export where atomization and gas-liquid mixing have good effects.
\end{abstract}

\section{Introduction}

With the development of science and technology, rare earth oxides are used widely, but the lack of the traditional production process for rare earth restricts the development of rare earth oxides. Meanwhile, China is a big country of reserving and producing rare earth, but the technology of producing and applying rare earth oxide is far behind that of the developed countries, showing the embarrassing situations that China is a country with rare earth's weak research and limited technology. Based on the situation above, Professor Wenyuan Wu proposed a new method of rare earth chloride spray pyrolysis step preparing solid spherical rare earth oxides, via direct thermal decomposition of rare earth chlorides to form oxides. This method has obtained patents. Compared to preparation method which commonly prepared rare earth oxides with our current rare earth carbonate or oxalate, the new method does not require precipitation, filtration, and other hightemperature calcination steps. It greatly reduces the production costs, waste, and emissions of acid [1,2]. For this process, research group designed Venturi spray pyrolysis reactor on the basis of thermodynamic calculations.

In this paper, we design a physical model in plexiglass material for the chloride pyrolysis reaction and have gotten valuable parameters by the physical model and numerical simulation, which provide strong basis for the manufacture of thermal state equipment and the determination of experimental conditions. Using the method of preparing rare earth oxides by direct thermal decomposition of rare earth chloride is expected to achieve green production of rare earth oxides and no emissions of waste gas and water, waste water discharges. Compared to the general stirred reactors, jet pyrolysis reactor has many advantages such as high reaction rate, short time, homogeneous reaction, good product quality, complete gas absorption, and no gas escaped phenomenon. Therefore, research group combines applications of jet pyrolysis technology in various industries and will apply pyrolysis technology to the production of rare earth oxides, hoping to find out an efficient, economic, and environmental friendly 
new technology which is suitable for production of rare earth oxides [3-5].

\section{Experimental Apparatus and Method}

The experimental device consists of air compressor, gasholder, home-grown spray pyrolysis device, reservoir, and cyclone separator. What we can see from Figure 1 is the homegrown spray pyrolysis device which we used in our experiment. During the experiment, because air stream is unstable when it was compressed by the air compressor, we need to connect a gasholder after the air compressor to stabilize the air flow. The air flow enters the Venturi tube from inlet and flows through the whole tube. In order to achieve the purpose of gas-liquid mixing, we connect the drainage tube between the center of throat and the lower reservoir. Because selfcitation amount of liquid is less, in order to achieve the twophase gas-liquid mixing and reacting more completely, it needs additional pressure of reservoir liquid surface, increasing the amount of liquid to enter. Therefore, the reservoir is designed to be in the form of seal and strong pressure.

Due to the structural properties of Venturi tube, when the continuous constant flow of fluid flows through a single Venturi tube, according to the continuity equation and Bernoulli's equation, the contraction of the fluid through the pipe section and the fluid flow area decreases; then the flow rate increases largely while pressure decreases, and a negative pressure at the center of the throat appears. Under certain experimental conditions, the liquid can be inhaled into the reactor from the lead. And when the throat diameter was smaller, the negative pressure and the amount of inhaled liquid were more significant. The resistance in the second half of the throat section of the Venturi tube was proportional to gas flowing. When the air flow or the diameter ratio was too large, under the effect of the resistance, the air flows into the storage tank through the drainage tube, and a large number of bubbles appeared in storage tank. When a large additional pressure of liquid is applied, the liquid was pushed into the Venturi tube hoses and then split to both sides. One of the streams was contrary to the flow direction of the air flow. The pressure of gas and additional liquid pressure interacted in the junction of tube; under certain experimental conditions, the pulsed spray phenomenon was achieved.

Based on the above discussion and aimed at different experimental purposes, 3 sets of venture tubes and drainage tubes were designed, respectively. 3 sets of Venturi tubes' inner diameter were $5 \mathrm{~mm}, 7 \mathrm{~mm}$ and $10 \mathrm{~mm}$, respectively; namely, the ratios were $20: 1,15: 1$, and $10: 1$. The inner diameters of the drainage tube were $3 \mathrm{~mm}, 5 \mathrm{~mm}$, and $7 \mathrm{~mm}$. The large diameter was $100 \mathrm{~mm}$, the constriction cone angle was $21^{\circ}$, and the extended segment of the tail cone angle was $15^{\circ}$. Venturi tube tail pipes' extended segment cone angle cannot be too large; otherwise the reflux reducing would reduce the efficiency of Venturi tube reactor. Venturi tube was connected to air tank using a pressure hose, and to ensure the stability of gas flow into the reducer section, the Venturi tube length of the inlet tube was designed to be $500 \mathrm{~mm}$. After the reaction, product collection device was cyclone.

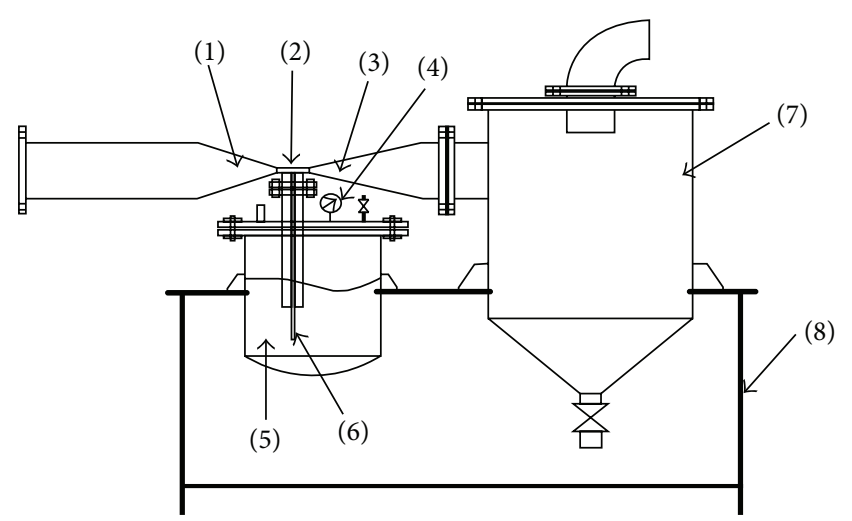
(1) Convergent section (inlet)
(5) Storage tank
(2) Center of throat
(6) Drainage tube
(3) Divergent section (outlet)
(7) Cyclone separator
(4) Piezometer
(8) Supporting frame

Figure 1: Home-grown spray pyrolysis device.

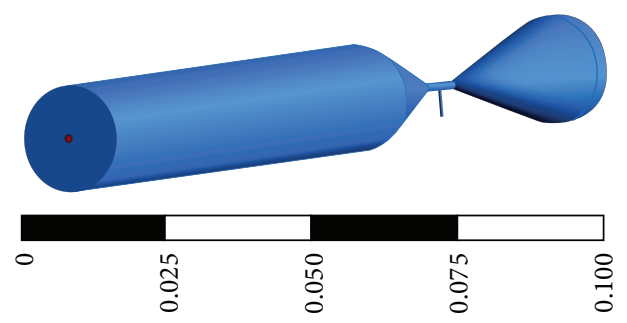

(m)

Figure 2: Jet reactor geometric model figure.

\section{Simulation Method}

At present, many researches on the improved jet reactor with venture tube were conducted with numerical simulation. In 2006, based on FLUENT software Wang et al. [6] and so forth applied the standard $\kappa-\varepsilon$ model and cavitation bubble dynamics model, the numerical simulation, on three different geometries, Venturi tube cavitation flow field was conducted, and the cavitation number in cavitation zone, pressure distribution, and gas holdup distribution were investigated. In 2007, Xie and Wu [7] and so forth conducted numerical simulation on gas-solid flow using two-fluid model, the interaction and flowing pattern of gas and solid phase were investigated, and the simulation results verified the accuracy of the model.

The commercial CFD software ANSYS FLUENT 12.0 was employed to investigate gas-liquid two-phase mixing process, flowing characteristics [8,9]. And Figure 2 showed us the three-dimensional jet reactor based on the real reactor in Figure 1.

3.1. Model of Jet Reactor. The model of jet reactor is as shown in Figure 2.

3.2. Partition in Network. In this paper, GAMBIT was employed to establish the geometric model and generate the mesh 
TABLE 1: Boundary conditions.

\begin{tabular}{lcccc}
\hline Boundary conditions & Air inlet & Liquid inlet & Wall & Outlet \\
\hline Styles & Velocity inlet & Pressure inlet & $\begin{array}{c}\text { Wall motion: stationary wall } \\
\text { Wall roughness: roughness constant } 0.5\end{array}$ & Pressure outlet \\
\hline
\end{tabular}

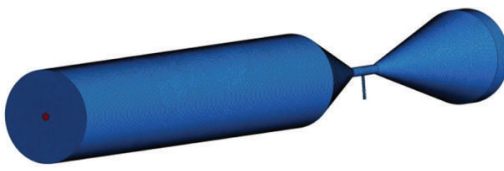

(a)



(b)
FIGURE 3: Grid sketch.

The grid quantity reached about 600,000 , and dense treatment was applied in throat. Because the grids are so dense we cannot see the grids clearly in Figure 3(a), but we can see the grids in the throat section of Venturi after amplification.

3.3. Equations. Mass conservation and momentum conservation equations could be written as follows $[10,11]$ :

$$
\begin{gathered}
\frac{\partial \rho}{\partial t}+\operatorname{div}(\rho U)=0 \\
\frac{\partial\left(\rho u_{i}\right)}{\partial t}+\operatorname{div}\left(\rho u_{i} U\right)=\operatorname{div}\left(\mu g r a d u_{i}\right)-\frac{\partial P}{\partial x_{i}}+S_{u_{i}} .
\end{gathered}
$$

Standard $\kappa-\varepsilon$ two-equation model equations were as follows:

$$
\begin{aligned}
& \rho \frac{\partial k}{\partial t}+\nabla\left(\rho U k-\left(\frac{\mu_{t}}{\sigma_{k}}\right) \nabla k\right)=\sum \alpha_{m}\left(G_{m}-\rho \varepsilon\right), \\
& \rho \frac{\partial \varepsilon}{\partial t}+\nabla\left(\rho U k-\left(\frac{\mu_{t}}{\sigma_{\varepsilon}}\right) \nabla \varepsilon\right) \\
& =\sum \alpha_{n}\left(C_{1} G_{n}-C_{2} \rho_{n} \varepsilon\right) \frac{\varepsilon}{k}
\end{aligned}
$$

Among them $\mu_{t}$ was the turbulent viscosity:

$$
\begin{aligned}
\varepsilon & =\frac{C_{D} k^{3 / 2}}{l}, \\
\mu_{t} & =\rho c_{\mu} \frac{k^{2}}{\varepsilon} .
\end{aligned}
$$

Model parameter values are $c_{1}=1.44, c_{2}=1.92, c_{\mu}=0.09$, $\sigma_{k}=1.0$, and $\sigma_{\varepsilon}=1.3$, where $c_{1}$ and $c_{2}$ appearing in (3) are fixed constants in software ANSYS FLUENT 12.0.

In this paper, the standard $\kappa-\varepsilon$ turbulence model coupled to VOF model was employed to simulate gas-liquid flow on steady three dimensions. The energy transfer, mass transfer, the influence of lifting force, and virtual mass force were ignored in this paper.

3.4. Boundary Conditions and Computing Methods. The pressure speed coupling PISO was used in separation algorithm.
The discretization schemes like momentum, turbulent kinetic energy, and turbulent dissipation rate all chose second-order upwind. The standard of convergence criteria was $10^{-3}$. The standard wall-function method was used to solve the area close to the wall. See Table 1.

\section{Results and Discussion}

4.1. Analysis of Concentration Field. The air inlet flow selected is $16.5 \mathrm{~m}^{3} / \mathrm{h}, 18.5 \mathrm{~m}^{3} / \mathrm{h}, 20 \mathrm{~m}^{3} / \mathrm{h}$, and $24 \mathrm{~m}^{3} / \mathrm{h}$. The inlet pressure of liquid sets is $0.01 \mathrm{MPa}$. The diameter of drainage tube is $3 \mathrm{~mm}$. Then the research begins for the gas-liquid twophase flow in the reactor.

It could be known that the negative pressure from air entering into the Venturi tube, the adsorption capacity, and the aqueous phase adsorbed into reactor all increase along with the increasing of air phase flow from Figure 4. The volume of aqueous phase in tail pipe under $20 \mathrm{~m}^{3} / \mathrm{h}$ is obviously higher than $16.5 \mathrm{~m}^{3} / \mathrm{h}$ and $18.5 \mathrm{~m}^{3} / \mathrm{h}$. The distribution of drops is also irregular. But the volume under $24 \mathrm{~m}^{3} / \mathrm{h}$ is lower than $20 \mathrm{~m}^{3} / \mathrm{h}$. A situation results that the overlarge air flow will reduce the standing time of the liquid phase in the reactor, which is adverse for the atomization effect formation of liquid phase particles even if it could absorb more liquid phases [12].

When the rate of flow chosen was $20 \mathrm{~m}^{3} / \mathrm{h}$ and the drainage tube diameter was $3 \mathrm{~mm}$, liquid inlet pressure values were $0 \mathrm{MPa}, 0.01 \mathrm{MPa}$, and $0.02 \mathrm{MPa}$; then we obtained the numerical simulation results for gas-liquid two-phase flow inside the reactor.

As we can see from Figure 5, with the increase of the liquid pressure, the liquid volume inside the reactor is increased. The volume fraction of the liquid under pressure $0.01 \mathrm{MPa}$ is significantly higher than $0 \mathrm{MPa}$, but when the pressure adds from $0.01 \mathrm{MPa}$ to $0.02 \mathrm{MPa}$, the effect of increasing the volume fraction of the liquid is not obvious. Liquid atomizes quickly under $0.01 \mathrm{MPa}$ and flows with the air blowing direction. And the gas-liquid two-phase fluid goes into hybrid adjustable pipeline; due to the increase of diameter, flow rate drops and spray liquid condenses into small droplets, converges at reactor by gravity along the pipeline, and ultimately flows out of the reactor.

If the rate of flow is $20 \mathrm{~m}^{3} / \mathrm{h}$, the liquid inlet pressure will be $0.004 \mathrm{MPa}$, and when the diameters of drainage pipe are $3 \mathrm{~mm}, 5 \mathrm{~mm}$, and $7 \mathrm{~mm}$, we got the numerical simulation results for gas-liquid two-phase flow inside the reactor.

It can be seen from Figure 6 that change of the drainage tube diameter has a great effect on water phase volume fraction at the bottom of Venturi tube divergent section. The increase of drainage pipe diameter will increase the capacity of the adsorbed water phase under unit pressure. When 

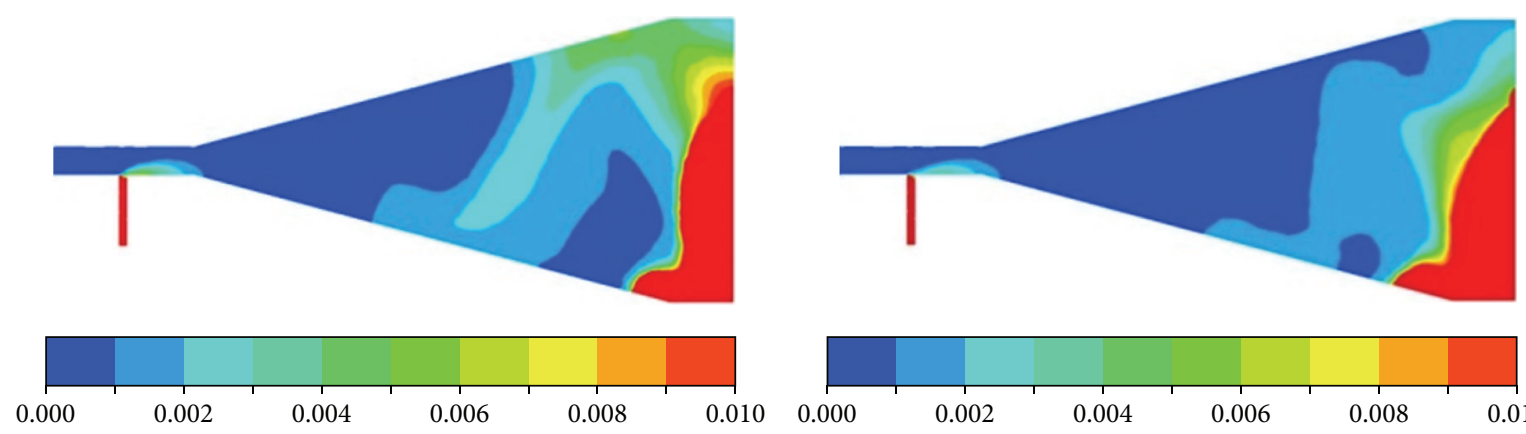

(a) $16.5 \mathrm{~m}^{3} / \mathrm{h}$

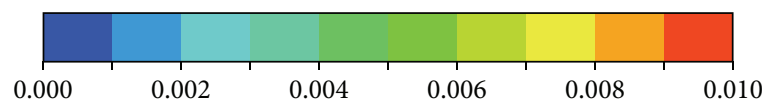

(b) $18.5 \mathrm{~m}^{3} / \mathrm{h}$
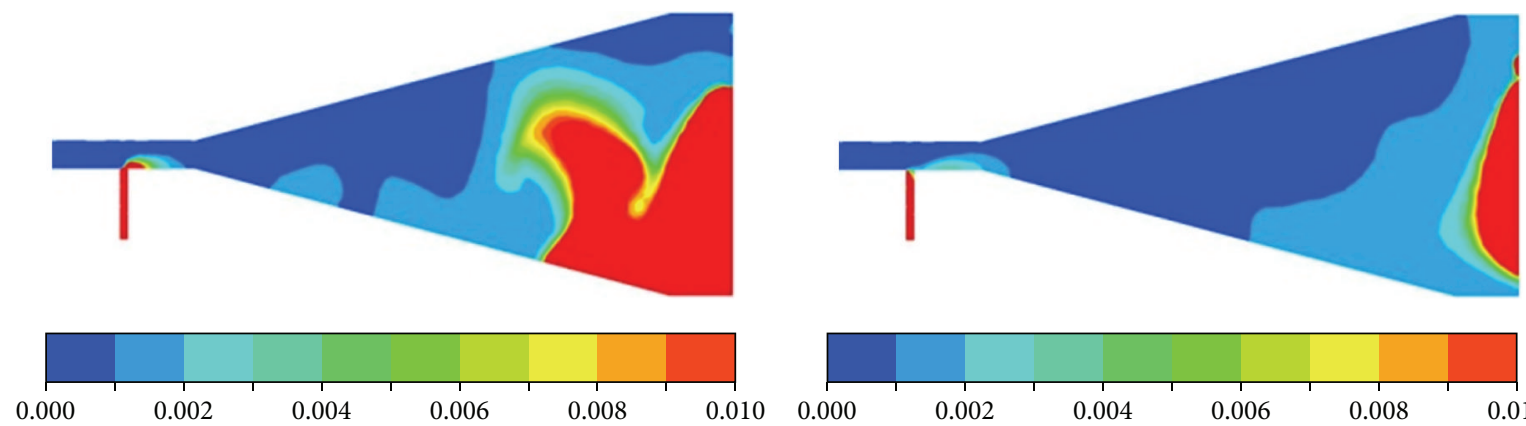

(c) $20 \mathrm{~m}^{3} / \mathrm{h}$

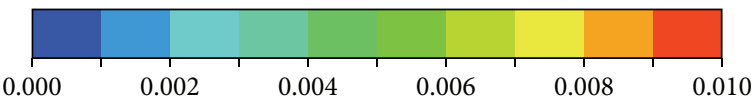

(d) $24 \mathrm{~m}^{3} / \mathrm{h}$

FIgURE 4: Water phase volume distribution under different air flow.
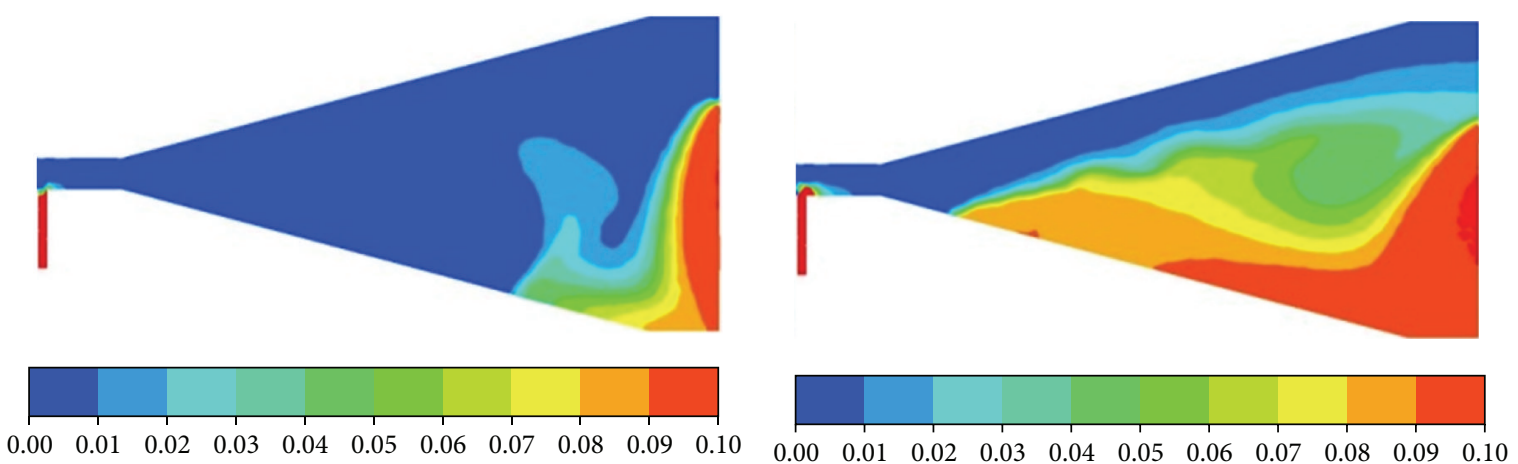

(a) $0 \mathrm{MPa}$

(b) $0.01 \mathrm{MPa}$



(c) $0.02 \mathrm{MPa}$

FIGURE 5: Water phase volume distribution under different pressure of liquid phase. 


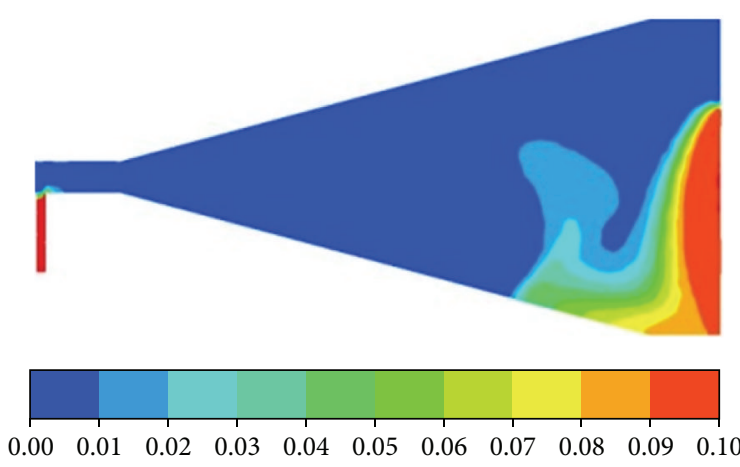

(a) $3 \mathrm{~mm}$
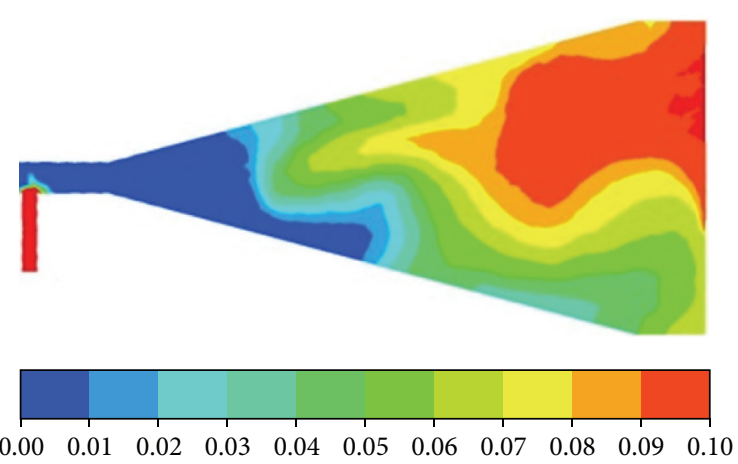

(b) $5 \mathrm{~mm}$
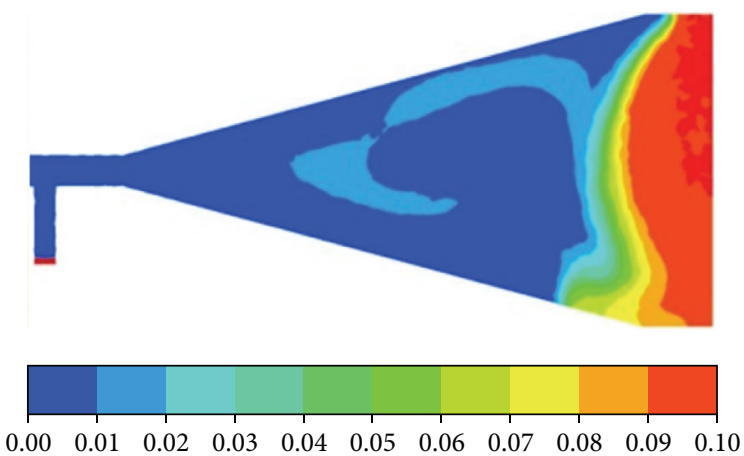

(c) $7 \mathrm{~mm}$

FIgURE 6: Water phase volume distribution under different drainage tube size.

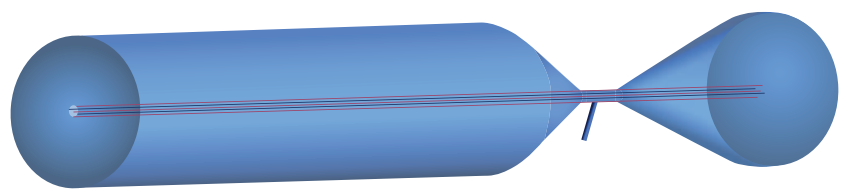

Figure 7: The choice of monitoring line.

the drainage pipe diameter is $5 \mathrm{~mm}$, the aqueous phase will spread all over the bottom of divergent section in the reactor, while the drainage pipe diameter is $7 \mathrm{~mm}$, the concentrated aqueous phase distribution is next to outlet, and the drainage tube aqueous phase concentrates at the entrance. It is indicated that larger drainage tube diameter will lead to more fluid into the reactor, which will offset the negative pressure produced by the air and reduce the adsorption capacity of the jet of the reactor, which is not conducive to the formation of the aqueous phase within the reactor in the atomization effect. In this case, the diameter of $5 \mathrm{~mm}$ is suitable.

4.2. Flow Field Analysis. It can be seen from Figure 7 that the monitoring line is located on the center axis, above the center axis, below the center axis, in front of the center axis, and behind the center axis. It is denoted as $y=0, y=4 \mathrm{~mm}$, $y=-4 \mathrm{~mm}, x=4 \mathrm{~mm}$, and $x=-4 \mathrm{~mm}$, and further analysis is made by monitoring the rate of change in the trend line speed inside the reactor [13].
It can be seen from Figure 8 that the highest speed of air blown into the reactor appeared at the initial position on the axis $y=0$. With the expansion of the tube, the speed decreased rapidly and tended to be stable. At the position of $z=0.6 \mathrm{~m}$, because of the shrinkage of the tube, the velocity of air flow increased quickly and fluctuated at the drainage tube with the change of water velocity. Behind the throat tube, because of the increase of the tube diameter, the velocity decreased rapidly and remained stable finally. The initial velocity on the other monitoring lines was zero. With the diffusion of air, the velocity increased rapidly and declined with the air movement. The velocity remained stable finally. At the throat tube, the velocity rapidly increased and fluctuated in the throat drainage tube. Through the variable diameter tube, the velocity decreased and became stable finally.

At the monitoring line $y=4 \mathrm{~mm}$ which was above the axis, because water flowed into the drainage tube and collided with air which produced a resultant force, the resultant velocity increased rapidly. However, the monitoring line of $y=-4 \mathrm{~mm}$ was below the axis, and at the drainage tube, the collision of the water and air offset the transverse velocity, which caused a significant decrease of the velocity.

It can be seen from Figure 9 that the $Y Z$ plane and $z=$ $10 \mathrm{~mm}, z=200 \mathrm{~mm}, z=505 \mathrm{~mm}, z=722 \mathrm{~mm}, z=840 \mathrm{~mm}$, and $z=862 \mathrm{~mm}$ were chosen as the monitoring positions.

It can be drawn from Figure 10 that the velocity was higher at the air inlet and in the Venturi tube. After air was blown into the reactor, the front tube remained stable, which indicated that the lengthening Venturi front tube caused 


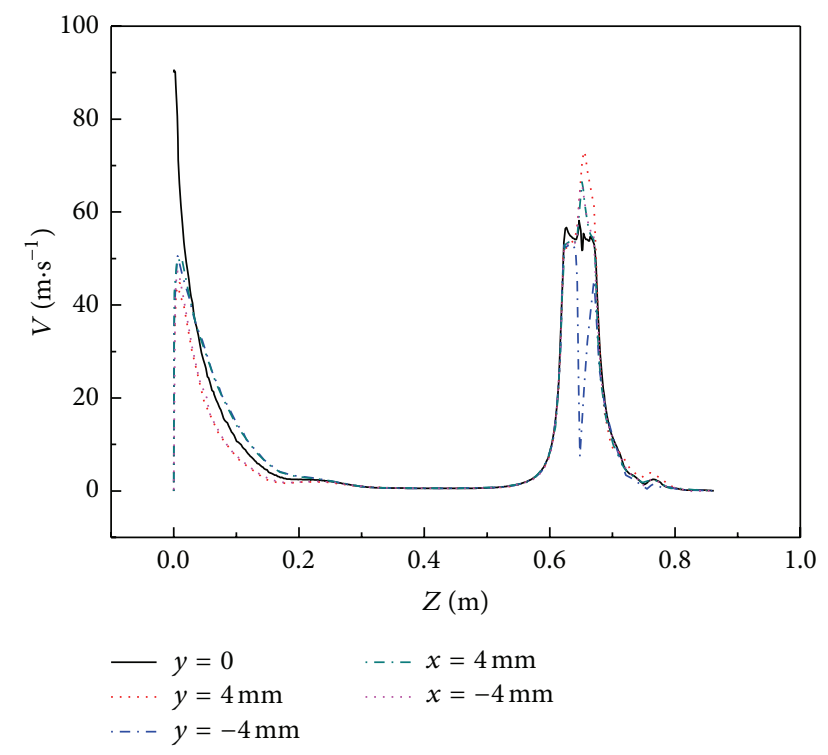

FIGURE 8: Rate trends.

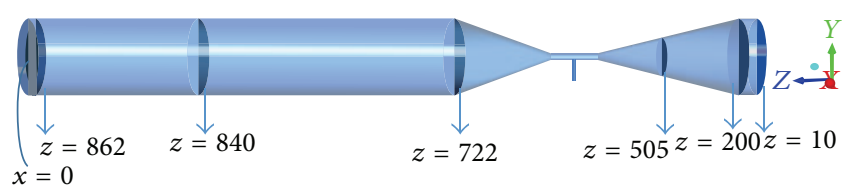

FIGURE 9: The choice of monitoring interface.

the stable air flow. With the shrinkage of the tube, the velocity of air flow increased rapidly in the Venturi tube, which caused negative pressure. The air became attached to the water and the droplet flowed into the Venturi tube from the drainage tube and then atomized. The droplet flowed to the back of the Venturi tube and because of the expansion of the tube section at the front part of the back tube, air velocity decreased. The atomized droplet dropped and collided with the lower edge of the back part of the Venturi tube, which accelerated the atomization. The fluid flowed along the higher edge with the levitation force and collision force and at the same time, there appeared an S-shape curve, which agreed well with the movement tendency in the experiment.

Figure 11 showed the fluid velocity vector on the different sections and the results were as follows. (a) At $z=10 \mathrm{~mm}$ section, because of the air blown into the center of jet reactor, the circulation appeared, which caused the change of the velocity vector. At the right side, with circulation collision with one another, the velocity decreased and the direction of the velocity vector was along the normal of the circulation. (b) At $z=200 \mathrm{~mm}$, with the increase of the gas injection distance in the front part of the Venturi tube, the velocity decreased. At the right side, the velocity vectors diffused from the collision center of circulation to the outward, which caused the change of the whole section. (c) At the section $z=505 \mathrm{~mm}$, the air flow remained stable, the velocity vectors gathered in the center of the section and because of the pressure, the center point of the velocity vectors shifted to the right side slightly. (d) At the $z=722 \mathrm{~mm}$ section, which was the center of

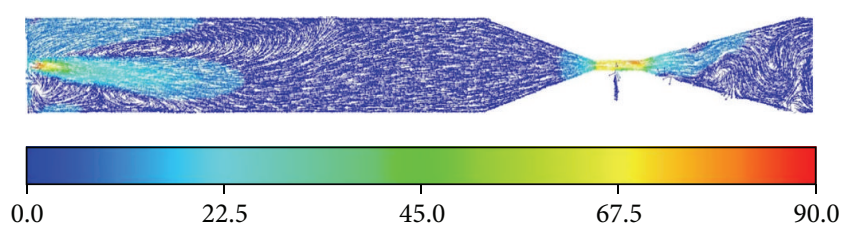

FIGURE 10: The diagram of fluid velocity vector in jet device.

the trail part of the Venturi tube, because of the change of tube diameter, the velocity of the mixture fluid of air and water decreased and there appeared two small vortices on both sides of the center. With the influence of atomization and the drainage force between two gas and liquid phases, the small vortex shifted to the upper right and because of the pressure, the vortex on the right side of the center was not formed. (e) When the cross-section is $z=840 \mathrm{~mm}$, this position is the junction between Venturi tube tail pipe and the straight pipe, so gas-liquid two-phase fluid flow here which affect the overall movement. Besides, it also has an influence on the export reflux pressure, making the upper half regional obvious reflow profile, so the bottom of the velocity vector trend goes downward. Due to hedge a significant role in the formation of the boundary curve, the normal direction is the circulation of hedge on the curve of the velocity vector direction. (f) At the plane of outlet $(z=862 \mathrm{~mm})$, most of the atomized droplets condensed, which make most of the velocity vector directed downward. While a part of atomization droplets is incondensable, the direction of velocity vector is upwards. It shows a distinct dividing curve on the plane of outlet. The curve gets a slight deviation to the right which is caused by press.

It can be seen from Figure 12 that the fluid had formed two vertical circulations at the front of Venturi tube; the radius of upper circulation is greater than that of the lower calculation; this is because the press in the reactor makes the air trajectory offset slightly below the horizontal line. At the middle part of the first half of venture reactor, air velocity is stable and the direction of air velocity vector turns right. Flowing with the pipe radius decreased, the air trajectory concentrates to the central axis of the Venturi reactor. At the other half of venture reactor, by the influence of atomization and liquid flow into first half of venture reactor, the fluid flows along upper wall. A vortex has been formed at the central part of the rear of Venturi tube, which will help gas-liquid phase fully contact in the reactor.

Figure 13 is the fluid trajectory figure in drainage tube for different inner diameter $(3 \mathrm{~mm}, 5 \mathrm{~mm}$, and $7 \mathrm{~mm})$. It can be seen that the fluid will have different trajectory with different drainage tube diameter. The bigger the diameter of drainage tube is, the bigger the liquid volume which flows into drainage tube will be at the same time; the internal pressure will be offset after atomizing, the angle between horizontal position and air trajectory will increase, and then the time of fluid turning to stable will be longer in the first half of venture reactor. (a) When the diameter of drainage tube is $3 \mathrm{~mm}$, the liquid phase and a part of atomizing air draught will flow along the wall, shaping a closed circulation in the center of reactor reducer. (b) When the diameter of drainage tube is 


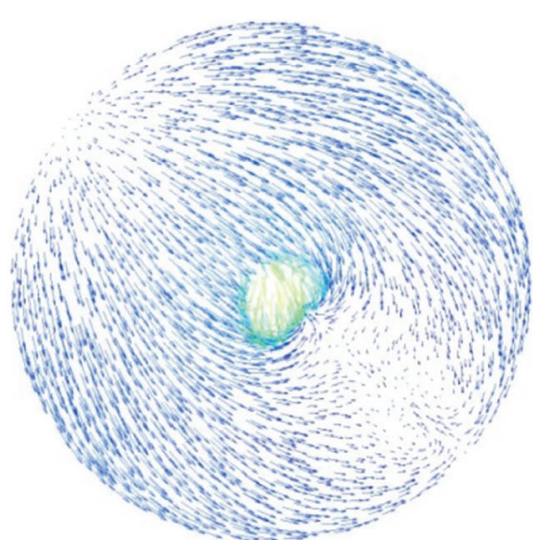

(a) $z=10 \mathrm{~mm}$

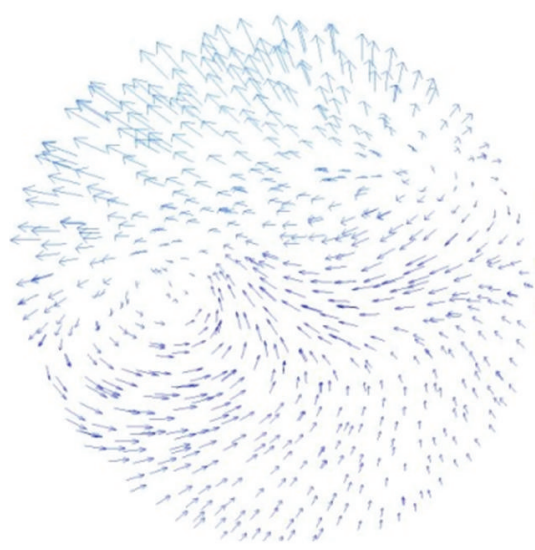

(d) $z=722 \mathrm{~mm}$

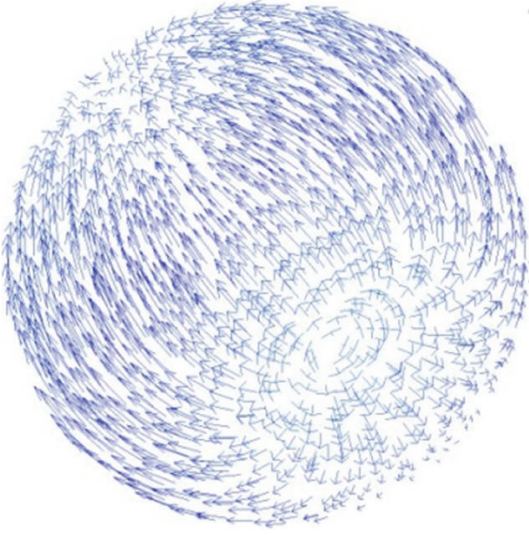

(b) $z=200 \mathrm{~mm}$

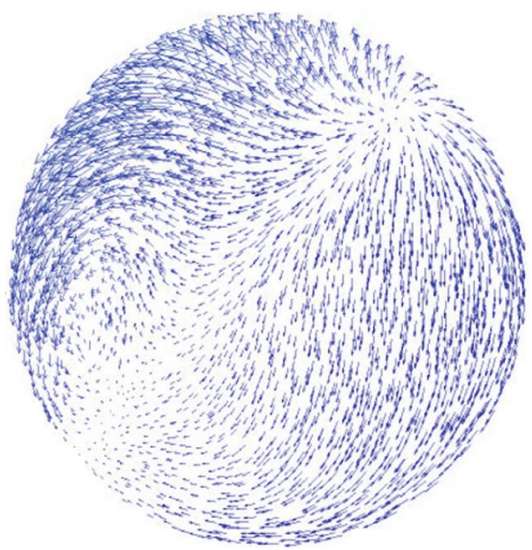

(e) $z=840 \mathrm{~mm}$

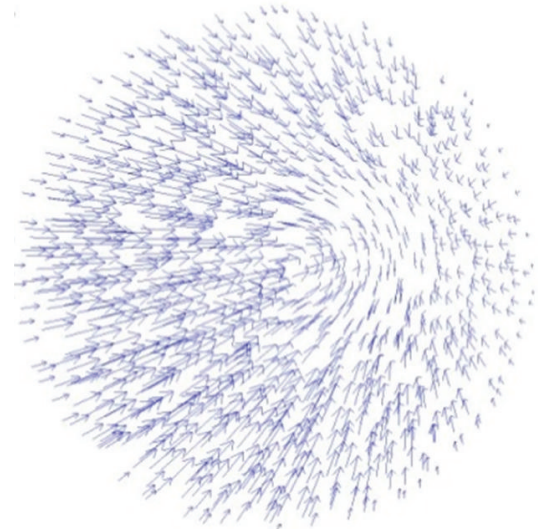

(c) $z=505 \mathrm{~mm}$

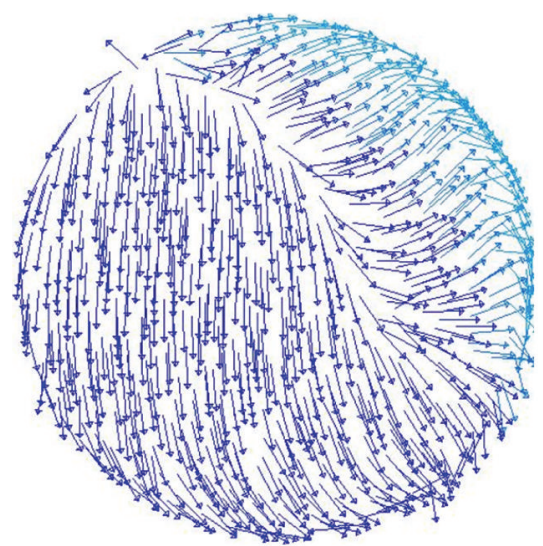

(f) $z=862 \mathrm{~mm}$

FIgURE 11: The diagram of fluid velocity vector on different sections.

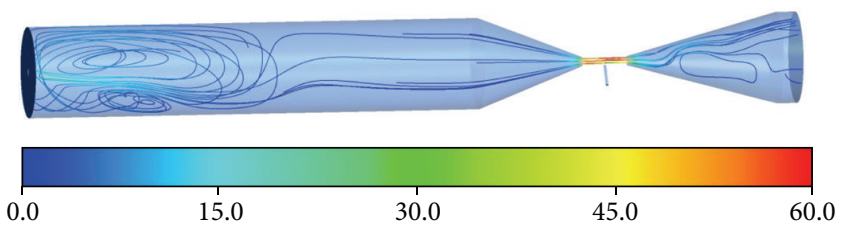

FIGURE 12: Picture of three-dimensional motion.

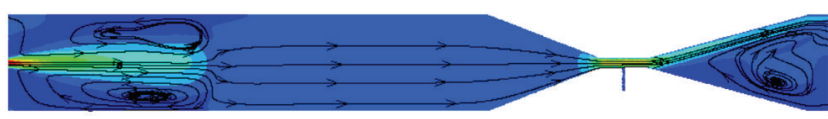

(a) The diameter of drainage tube: $3 \mathrm{~mm}$

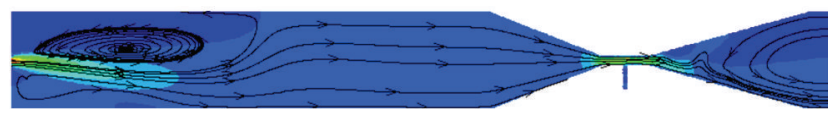

(b) The diameter of drainage tube: $5 \mathrm{~mm}$

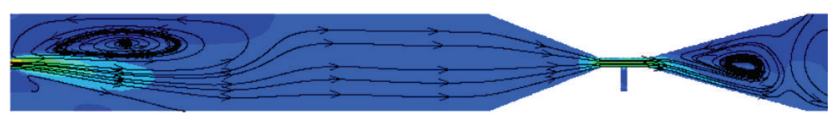

(c) The diameter of drainage tube: $7 \mathrm{~mm}$

FIGURE 13: Picture of fluid motion.
$5 \mathrm{~mm}$ and the liquid volume is moderate, which goes through drainage tube into reactor, the atomized droplet converges gradually with the change of gas velocity and pressure. Part of the fluid outflows from the bottom wall of reducer along the reactor and shaped a big back flow in the exit. (c) There will be a closed circulation in the center of drainage tube and a backflow near the exit.

\section{Conclusions}

By numerical simulation of jet reactor, the characteristics of fluid flow in jet reactor are analyzed, and the conclusions are made as follows.

Concentration field analysis: when drainage pipe diameter is $3 \mathrm{~mm}$, the additional pressure at the inlet of liquid phase is $0 \mathrm{MPa}$ and $20 \mathrm{~m}^{3} / \mathrm{h}$ of air inlet flow rate is more suitable for pyrolysis reaction; when air flow tube diameter is $3 \mathrm{~mm}$, air inlet flow rate is $20 \mathrm{~m}^{3} / \mathrm{h}$ and $0.01 \mathrm{MPa}$ of the liquid inlet additional pressure is more appropriate; for air inlet flow rate $20 \mathrm{~m}^{3} / \mathrm{h}$ and the liquid inlet additional pressure $0 \mathrm{MPa}$, choosing drainage tube of $5 \mathrm{~mm}$ diameter is more appropriate; such conditions can guarantee that the gas-liquid twophase distribution at the tail region of Venturi tube is more 
uniform, and it is easy to get a good mixed effect for gas-liquid two-phase flow in the reactor.

Flow field analysis: from the analysis of speed of horizontal straight line and longitudinal of the inside jets reactor, and with the comprehensive analysis of the fluid trajectories of the reactor, we found that the fluid speed decreased gradually at the front of Venturi reactors and reached stability at the middle position of Venturi tube gradually. It is shown that elongating the length of the front part of Venturi pipe is conducive to obtain a stable air flow. At the rear part of reducer pipe of Venturi, the fluid can form a closed loop; the outlet generated backflow; in this situation, the gas-liquid two-phase residence time will increase at tail pipes, atomization and mixing will be better, and the fluid flows out of the reactor in a track of fluctuating curve type forward; they have some good results compared with experiments and simulation results.

\section{Conflict of Interests}

The authors declare that there is no conflict of interests regarding the publication of this paper.

\section{Acknowledgments}

This work was supported by National Natural Science Foundation of China (no. U1202274, 51004033), National Science \& Technology Support Program (no. 2012BAE01B02), and National High-Tech R\&D Program of China (863 Program no. 2012AA062303).

\section{References}

[1] X. Y. Zhao, Y. Zhang, H. C. Gu, and W. Gao, "Synthesis of rare earth ultrafine powders by spray pyrolysis(I)-particle morphology and formation mechanism of $\mathrm{Y}_{2} \mathrm{O}_{3}$ powder," Chemical Journal of Chinese Universities, vol. 4, pp. 16-19, 1998.

[2] Z. Y. Chen, D. J. Zhang, and Y. T. Qian, "Powder prepared by spray pyrolysis method and its application," Bulletin of the Chinese Ceramic Society, vol. 6, pp. 54-62, 1998.

[3] Z. P. Liu and X. T. Wang, "Progress in study of rare earth compound micro powders producing methods," Chinese Rare Earths, vol. 6, pp. 68-73, 2003.

[4] X. M. Dai, "Spray pyrolysis-a kind of important micro powder preparation technology," Powder Technology, vol. 2, pp. 28-33, 1995.

[5] L. S. Jian and T. H. Zhang, "Adjusting uneven brightness of spray liquid particle image," Computer Engineering, vol. 7, pp. 180-181, 2006.

[6] Z. Y. Wang, X. D. Zhang, and H. Z. Yang, "Numerical simulation of cavitation flow field in the venturi," Computers and Applied Chemistry, vol. 23, no. 10, pp. 939-942, 2006.

[7] F. Xie and Z. S. Wu, "Numerical simulation and experimental study of gas-solid two-phase flow in venturi tubes," Journal of Power Engineering, vol. 27, no. 4, pp. 237-241, 2007.

[8] P. Shao, T.-A. Zhang, Z. Zhang, and Y. Liu, "Numerical simulation on gas-liquid flow in mechanical-gas injection coupled stirred system," ISIJ International, vol. 54, no. 7, pp. 1507-1516, 2014.
[9] A. Almaraz, C. López, I. Arellano et al., "CFD modelling of fluid flow in a Peirce-Smith converter with more than one injection point," Minerals Engineering, vol. 56, pp. 102-108, 2014.

[10] L. Zhang, Y. Guo, and S. He, "Numerical simulation of threedimensional incompressible fluid in a box flow passage considering fluid-structure interaction by differential quadrature method," Applied Mathematical Modelling, vol. 31, no. 9, pp. 2034-2049, 2007.

[11] M. J. Zhang, H. Z. Wang, H. Z. Gu, and A. Huang, "Numerical simulation of molten steel flow in argon bottom blown tundish," Journal of Iron and Steel Research, vol. 19, no. 2, pp. 16-19, 2007.

[12] N. Tang, H. J. Zhang, and W. Wang, "Computational fluid dynamics numerical simulation of vacuum membrane distillation for aqueous $\mathrm{NaCl}$ solution," Desalination, vol. 274, no. 1-3, pp. 120-129, 2011.

[13] Y.-C. Huang, J. Tang, Q.-R. Xie, and Y.-F. Ma, "Application of computational fluid dynamics in chemical engineering," Modern Chemical Industry, vol. 27, no. 5, pp. 65-68, 2007. 


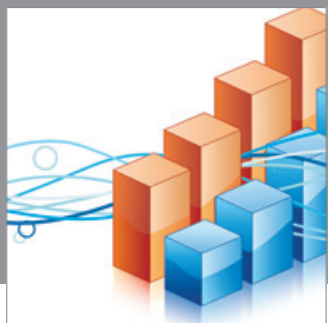

Advances in

Operations Research

mansans

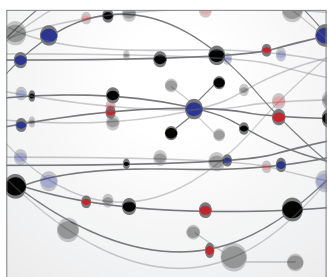

The Scientific World Journal
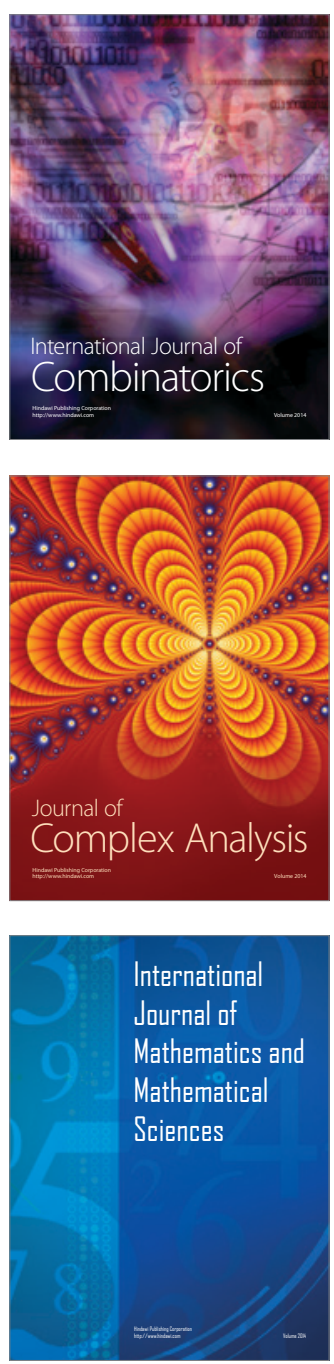
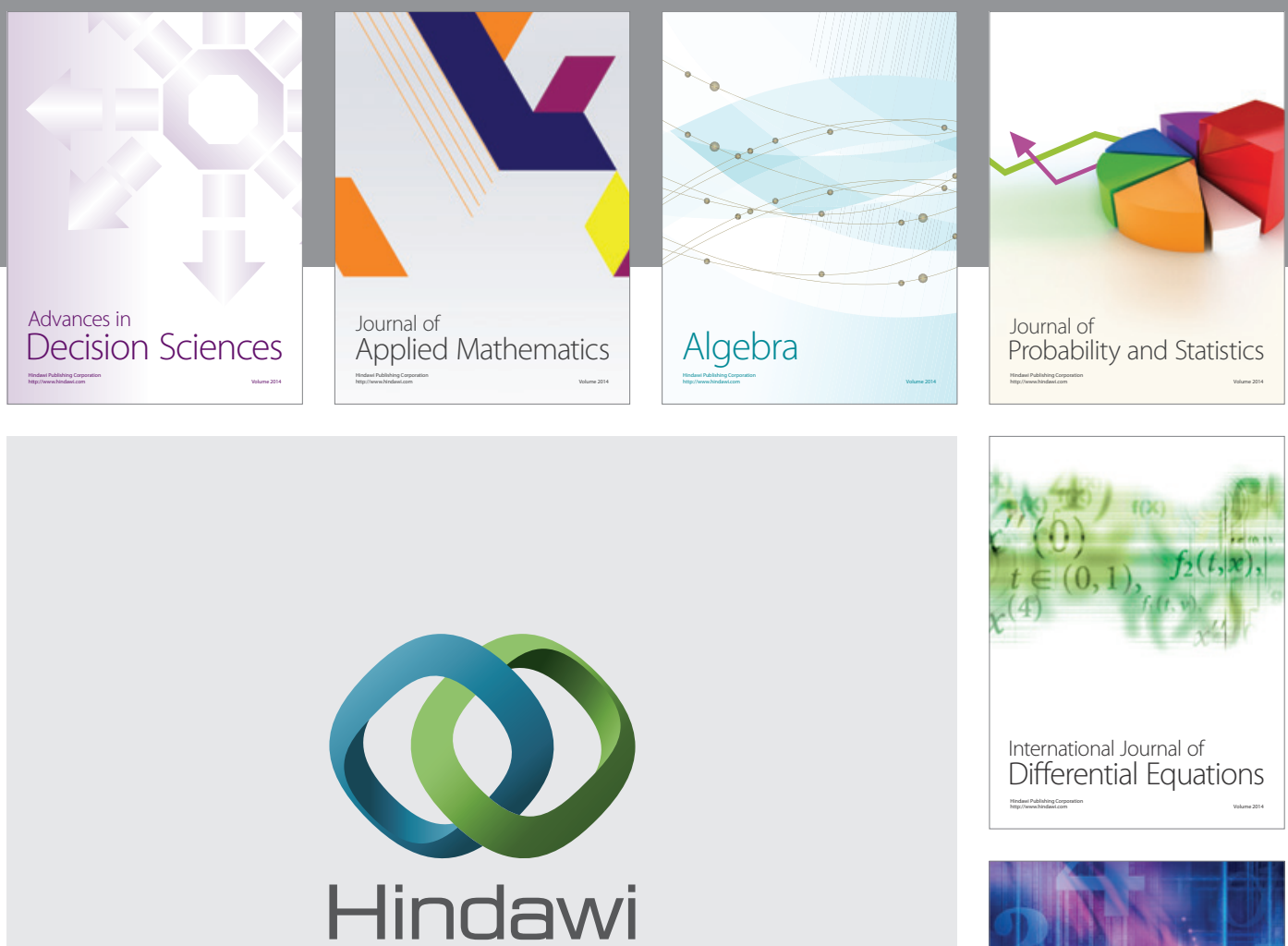

Submit your manuscripts at http://www.hindawi.com
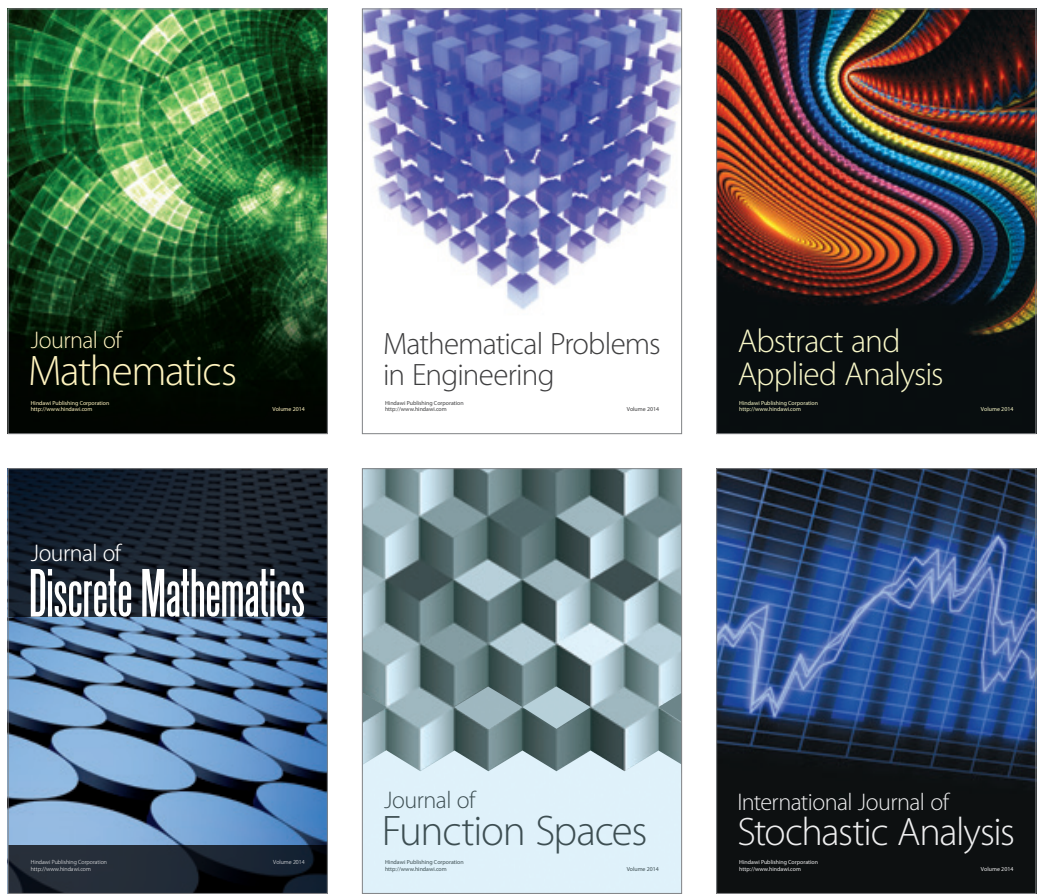

Journal of

Function Spaces

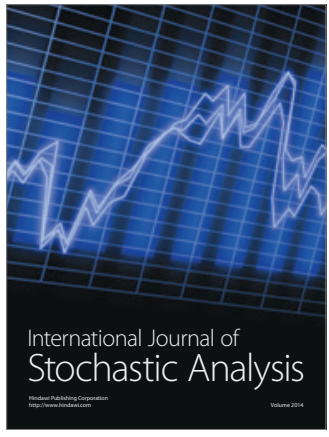

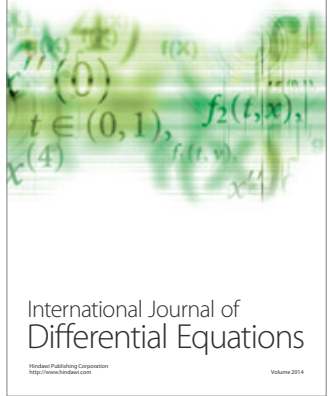
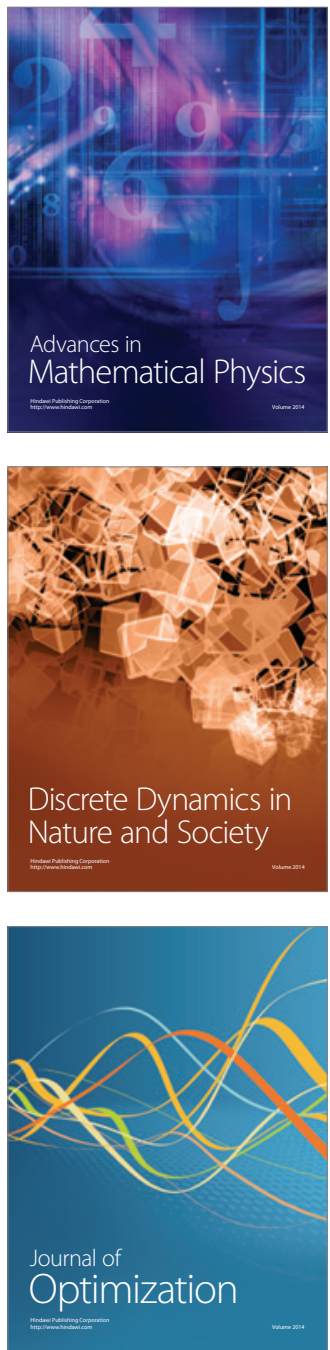